\title{
Does Type 5 of Takayasu's Arteritis Require Subclassification?
}

\author{
Sandeep Moode ${ }^{1}$ Suneethi Malayathi ${ }^{2} \quad$ Jyotsna Maddury ${ }^{1} \quad$ Lalita Nemani $^{1}$
}

\author{
${ }^{1}$ Department of Cardiology, NIMS, Hyderabad, Telangana, India \\ 2Department of Radiology, CARE Hospital, Hyderabad, \\ Telangana, India \\ Indian J Cardiovasc Dis Women-WINCARS 2018;3:23-26
}

\begin{abstract}
Address for correspondence Sandeep Moode, DM, Department of Cardiology, NIMS, Hyderabad, Telangana, India

(e-mail: Sandeep.moode@gmail.com).
\end{abstract}

\begin{abstract}
Background Takayasu's arteritis (TA) is an inflammatory vascular disease of the young involving the large elastic arteries resulting in occlusive or ectatic changes, mainly in the aorta and its major branches as well as the pulmonary artery and its branches.

Materials and Methods The authors reviewed the TA patients admitted over 2 years in their department and who underwent catheterization. They noted the clinical presentations, two-dimensional (2D) echocardiographic features, and angiogram details of all these patients.

Results The study population constituted 24 patients with mean age of 28 years. Out of them, 21 (87.5\%) were females. Most of the admitted TA patients presented with claudication complaints (79.2\%), 37.5\% patients had breathlessness, and $12.5 \%$ patients had typical stable angina symptoms. Five patients had 2D echo abnormalities, and rest of the patients had normal echocardiogram. Coronary angiogram (CAG) along with aortogram and selective peripheral arteriograms were performed of all these patients. Left main coronary artery (LMCA) disease was seen in three (12.5\%) patients. Case details of two (8.3\%) patients with multiple anomalous communications of the coronaries with pulmonary vasculature were described.

Conclusion TA patients who came for catheterization were predominantly females, mainly involving the aorta and its main branches. In the authors' TA series of patients,

Keywords

- Takayasu's arteritis

- coronary involvement

- subclassification type 5 presentation was there in five $(20.8 \%)$ patients (three cases of LMCA + two cases of coronary artery to pulmonary parenchymal fistulas). They propose to subclassify type 5 of TA, as they report coronary artery to pulmonary parenchymal fistulas along with the previous literature.
\end{abstract}

\section{Introduction}

Takayasu's arteritis (TA) is a chronic systemic inflammatory disease of the young predominantly involving the large elastic arteries. This inflammation may result in stenosis (even occlusive lesions) or ectatic changes mainly in the aorta and its major branches. ${ }^{1,2}$ The involvement of the coronary artery is rare and is classified as type 5 of TA by Panja et al. ${ }^{3}$ Previously, isolated cases of TA associated with coronary fistulas were reported by Horimoto et al ${ }^{4}$ and Ercan et al. ${ }^{5}$

Coronary arteriovenous fistulas (CAVFs) etiology can be congenital or acquired and are quite rare. They can arise from one of the coronary artery and drain into any of the cardiac chambers and/or great vessels. ${ }^{6}$ Rarely coronary arteries can give collateral circulation to lung parenchyma in pulmonary atresia. However, coronaries giving collaterals to lung parenchyma without pulmonary atresia or tetralogy of Fallot (TOF) is less reported.

\section{Materials and Methods}

\section{Study Population}

We retrospectively reviewed the TA patients fulfilling the American College of Rheumatology classification criteria for 
$\mathrm{TA},{ }^{7}$ who were admitted for catheterization between January 2015 and 2017 December in cardiology department of our institute. Informed consent for cardiac catheterization and angiogram were taken from the patients. We excluded the patients who did not meet American College of Rheumatology classification criteria for TA and in whom coronary angiogram (CAG) was not done.

\section{Tools and Techniques}

We noted the clinical presentations, two-dimensional (2D) echocardiographic features, and angiogram details of all these patients.

\section{Results}

Study population constituted approximately 24 patients with mean age of $28 \pm 3$ years. Most of the study population constituted female patients, with 21 out of the 24 patients being female (87.5\%) and the rest 3 male (12.5\%). Out of 24 admitted TA patients, 19 (79.2\%) patients presented with claudication complaints, predominantly upper limb claudication. Nine (37.5\%) patients had breathlessness, with class 2 symptoms in seven and class 3 or 4 in two patients. Three (12.5\%) patients had typical stable angina symptoms, but this history was elicited after finding lesion on CAG. Demographic and clinical presentation of male and female patients is shown in - Table $\mathbf{1}$.

Echocardiogram showed regional wall motion abnormality in the left anterior descending artery territory, with preserved left ventricular function being noted in $2(8.3 \%)$ cases, and three (12.5\%) patients had global hypokinesia with moderate to severe left ventricular (LV) dysfunction and the rest of patients had normal echocardiogram.

CAG was performed along with aortogram and selective peripheral arteriograms of all these patients. We noted that three (12.5\%) patients had left main coronary artery (LMCA) disease and four had mild left descending artery disease. Two patients had anomalous communication of coronaries with pulmonary vasculature. Rest of the patients had normal coronaries.

The details of these two cases of TA with coronary artery fistula to pulmonary parenchyma were described.

Case 1: A 27-year-old male patient was admitted with complaints of giddiness. There were no complaints of chest pain. On examination the patient had decrease in bilateral carotids, brachial and radial artery pulsations with pulse rate of 86 beats/min, and blood pressure of 180/100 mm Hg.

On investigation, hemoglobin was $14 \mathrm{~g} / \mathrm{dL}$ and total leucocyte count was $19,300 / \mathrm{mm}^{3}$. Complete urine examination was normal with blood urea of $16 \mathrm{mg} / \mathrm{dL}$ and serum creatinine of $0.8 \mathrm{mg} / \mathrm{dL}$. The erythrocyte sedimentation rate (ESR) $(66 \mathrm{~mm} / \mathrm{h})$ and C-reactive protein (CRP) $(12 \mathrm{mg} / \mathrm{L})$ values were higher. Electrocardiogram (ECG) and chest radiography were normal. Echocardiography showed no regional wall motion abnormality and good biventricular function. In view of increase in inflammatory markers, we discussed with the rheumatologist to control the disease activity before taking for catheterization.

Arterial Doppler ultrasound of right upper limb showed reduced caliber, irregular lumen, and partial color uptake with biphasic spectral pattern and reduced peak systolic velocities. Altered flow with reduced velocities in left upper limb and bilateral lower limbs was seen. Diffuse intimal thickness was noted measuring $2.4 \mathrm{~mm}$ at the proximal subclavian artery. Computed tomographic (CT) angiography showed diffuse circumferential mural thickening involving ascending and arch of the aorta and evidence of diffuse mural thickening with mild stenosis of the brachiocephalic artery and severe stenosis of the right common carotid artery and right subclavian artery. Short-segment severe stenosis of the proximal left common carotid artery and long-segment severe stenosis of the left subclavian artery were noted with minimal mural thickening of the descending thoracic aorta. There was diffuse circumferential mural thickening involving the abdominal aorta extending into the celiac trunk and superior and inferior mesenteric and bilateral renal arteries. Bifurcation of the aorta and bilateral common, external iliac arteries were normal course and caliber.

On angiogram, the right carotid is diffusely diseased with focal $90 \%$ narrowing in the right common carotid artery, left common carotid proximal long-segment $70 \%$ stenosis, right subclavian proximal $80 \%$ stenosis, and left subclavian $60 \%$ stenosis. Renal angiogram showed bilateral ostioproximal $80 \%$ stenosis.

CAG showed normal epicardial coronary arteries with multiple coronary to pulmonary parenchymal fistulas. These fistulas or anomalous vessels originate from the proximal right coronary and left circumflex coronary arteries, communicating with lung parenchyma directly through a network of vessels (-Figs. 1, 2).

Case 2: A female patient aged 35 years presented with exertional breathlessness and giddiness. She had no complaints of chest pain, palpitations, or syncope.

On examination the patient was conscious and coherent, and vital signs were normal. She had carotid bruise and decreased pulsations of bilateral carotids and brachial and radial artery.

On investigations hemoglobin was $12.6 \mathrm{mg} / \mathrm{dL}$, total leucocyte count was $11,000 / \mathrm{mm}^{3}$, ESR was $52 \mathrm{~mm} / \mathrm{h}$, and CRP

Table 1 Demographic and clinical presentation of the study population

\begin{tabular}{|l|l|l|l|}
\hline Symptoms & Total $(\boldsymbol{n}=\mathbf{2 4 )}$ & Females $(\boldsymbol{n}=\mathbf{2 1})$ & Males $(\boldsymbol{n}=\mathbf{3})$ \\
\hline Claudication and other noncardiac symptoms & $19(79.2 \%)$ & $17(89.5 \%)$ & $2(10.5 \%)$ \\
\hline Breathlessness & $9(37.5 \%)$ & $7(77.7 \%)$ & $2(22.2 \%)$ \\
\hline Typical stable angina & $3(12.5 \%)$ & $2(66.6 \%)$ & $1(33.3 \%)$ \\
\hline
\end{tabular}




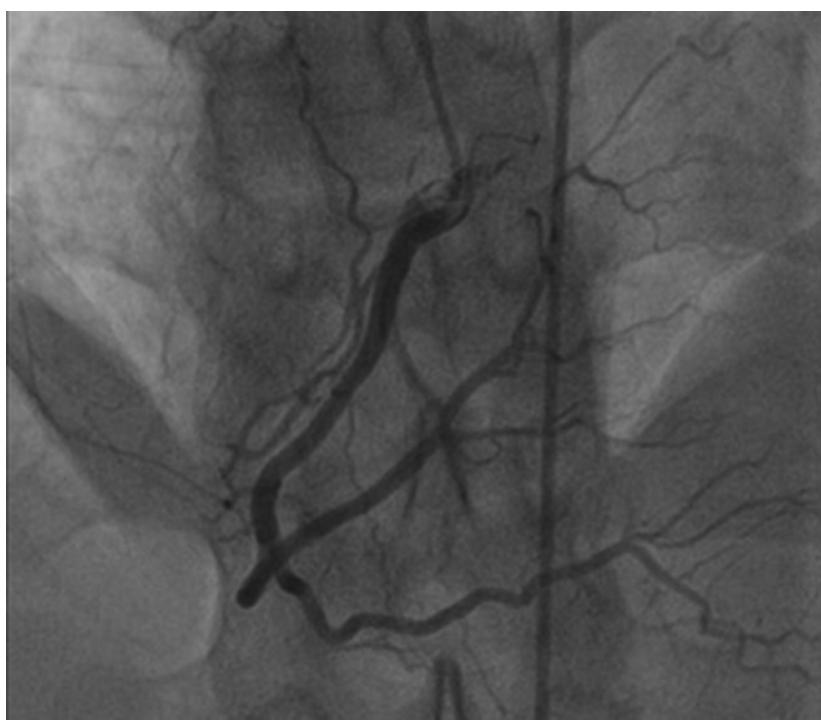

Fig. 1 Anomalous vessels originating from the proximal right coronary arteries, draining into the pulmonary parenchyma.

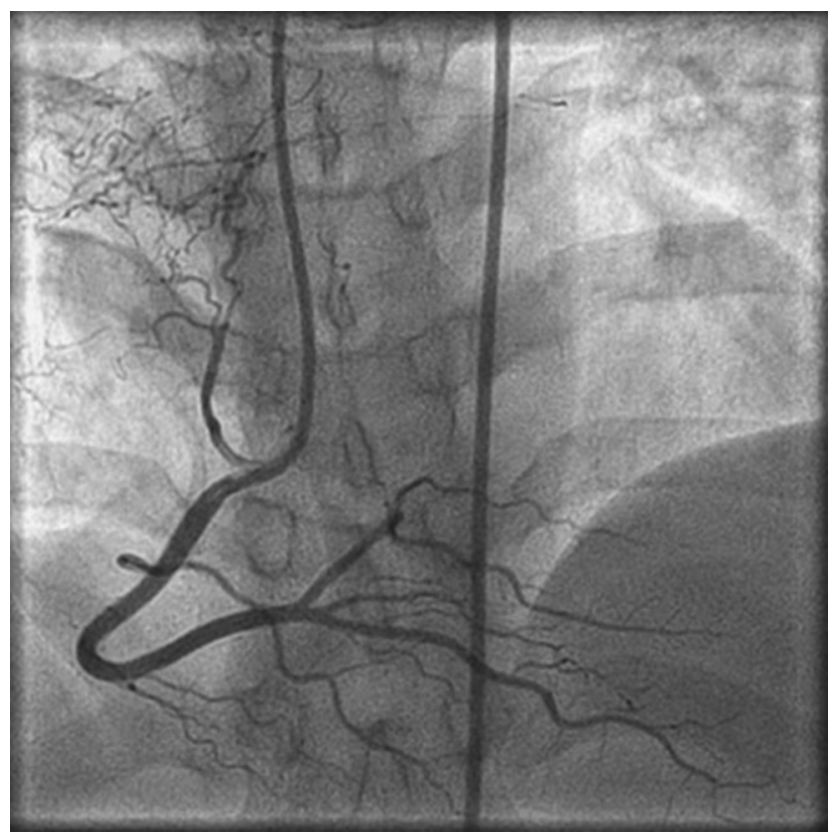

Fig. 3 Right coronary artery to pulmonary parenchyma.

was $14 \mathrm{mg} / \mathrm{L}$. On angiogram the left common carotid was diffusely diseased with proximal long-segment $80 \%$ stenosis. Left external carotid showed ostioproximal 70\% stenosis. Renal angiogram was normal.

CAG was normal with multiple coronary artery fistulas showing fistulous communication of sinus node artery of the right coronary artery (RCA), with pulmonary parenchyma of the right middle lobe of the lung (-Fig. 3) and two smallcaliber vessels arising one from the left main coronary and another from the proximal left anterior descending, which were in communication with lung parenchyma ( - Fig. 4).

\section{Discussion}

Involvement of the aorta and its main branches is well known in TA patients. Even though the coronary involvement is

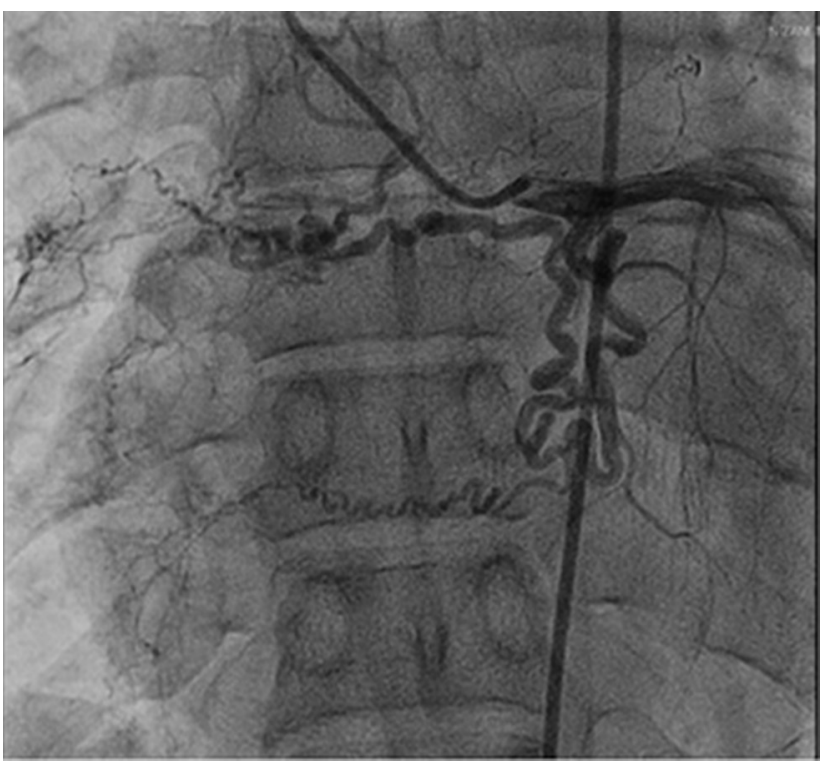

Fig. 2 Left circumflex artery to pulmonary parenchymal communication.

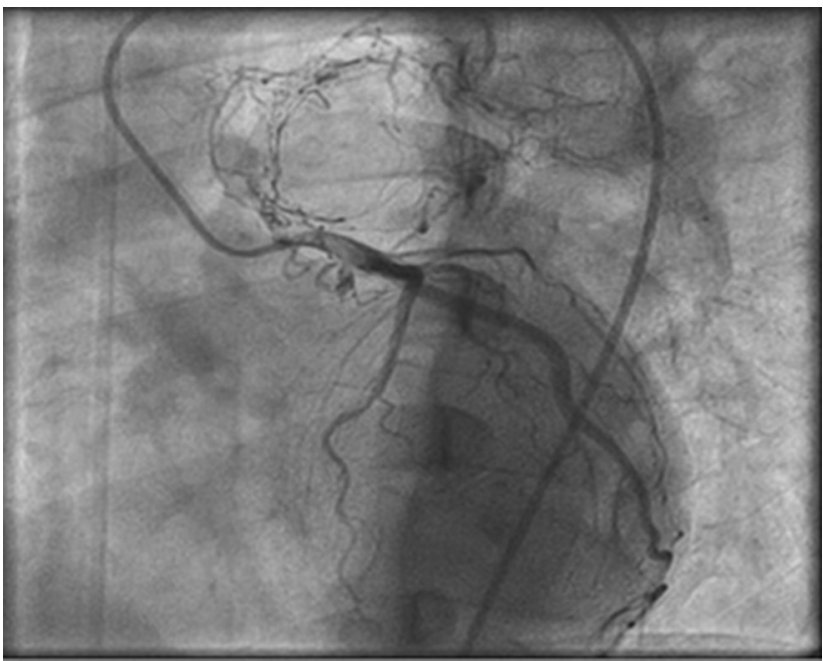

Fig. 4 Anomalous vessels arising left main coronary and from proximal left anterior descending to the lung parenchyma.

reported previously and classified this entity as type 5 of TA, its incidence is rare. We report two cases of TA having multiple coronary to pulmonary communications.

The anomalous vessel from the coronary artery may communicate with the lumen of a cardiac chamber (right ventricle $41 \%$, right atrium $26 \%$, left atrium $5 \%$, left ventricle $3 \%$ ), coronary sinus ( $7 \%)$, superior vena cava (SVC) (1\%), pulmonary artery (17\%), or the pulmonary vein. Approximately $90 \%$ of venous drainage is seen into the systemic venous side. ${ }^{8}$ The RCA is involved in fistulous formation (50\% of cases) and is often symptomatic. Left coronary artery (LCA) is involved in about $42 \%$ of fistulas and is often asymptomatic. Both RCA and LCA are involved in $5 \%$ of cases. ${ }^{9}$ Usually coronary fistulas are single, but multiple and complex fistulas have been reported. Cardiovascular anomalies are associated in 5 to $30 \%$ of cases. ${ }^{10}$

Systemic-pulmonary shunts in TA known to occur. The Asian population has more TA, but the pulmonary artery involvement does not have any racial or geographic predilection. ${ }^{11}$ 
In our first case of male TA patient, one angiogram showed anomalous vessels originating from the proximal right coronary and left circumflex coronary arteries, communicating with lung parenchyma through a network of vessels.

In our second case of female patient, there was a fistulous communication of sinus node artery of RCA with lung parenchyma of right middle lobe, which was similar to case report by Horimoto et al. ${ }^{4}$ In the study by Horimoto et al, ${ }^{4} \mathrm{RCA}$ branch giving fistula emptied into the proximal right pulmonary artery and the distal branches of the middle lobe artery.

In addition to RCA to pulmonary parenchymal communications, our female patient had two small-caliber vessels arising one from the left main coronary and another from the proximal left anterior descending, which were in communication with pulmonary vasculature supplying lung parenchyma. Furthermore, this patient had three coronary to pulmonary fistulas, which was similar in having multiple coronary to pulmonary communications in the case report done by Ercan et $a 1,{ }^{5}$ who found five coronary to pulmonary, seven bronchopulmonary, and two other systemicpulmonary shunts in a 37-year-old female TA patient.

Yamato et $\mathrm{al}^{12}$ mentioned there was no predilection of the vascular involvement for any side of the lung. Lupi et $\mathrm{al}^{13}$ have indicated that the right pulmonary artery and its lobar and segmental branches were most often affected, which was correlated with our case report. Additionally, segmental or subsegmental branches were less commonly involved than lobar branches; the upper branches were more commonly affected than the basal branches.

Karabay et a ${ }^{14}$ presented a 35 -year-old woman who was diagnosed with TA 15 years ago. She was hospitalized because of New York Heart Association class 2 heart failure and during conventional invasive coronary angiography, coronary fistulas from the RCA, left anterior descending, and ramus intermedius artery to the bronchial arteries were noted.

Coronary artery involvement is mentioned as type 5 of TA previously. However, all the cases reported were epicardial arterial stenosis or occlusions, but not mentioned about other anomalies of coronary arteries. Literature research, along with our two cases of coronary artery to lung collaterals, gives an idea that this anomaly also should include in the type 5 variant or subclass of type 5 . Therefore, we propose the subclassification of type 5 of TA as:

\section{Type $5 \mathrm{~A}$ obstructive coronary artery disease (Type 5A- OCAD)}

2. Type $5 B$ coronary artery to pulmonary fistulas (Type 5BCAPF), depending on the number of feeding coronary arteries into two subtypes

i. Single coronary artery giving lung parenchymal collaterals

ii. More than one coronary artery giving lung parenchymal collaterals

\section{Conclusion}

Takayasu's arteritis mainly involves the aorta and its main branches. The involvement of the coronary artery is rare, which was previously classified as type 5 . We report two cases of TA in which there was communication from coronary arteries to lung parenchyma without any pulmonary atresia. In previous studies, we mainly discussed about the obstructive coronary artery disease, especially ostial coronary artery involvement, but only case reports were there for this type of coronary collaterals. In our TA series of patients, type 5 presentation was there in five (20.8\%) patients (three cases of LMCA + two cases of coronary artery to pulmonary parenchymal fistulas). Along with the previous reported cases, we want to propose subclassification of type 5 TA.

\section{Conflict of Interest}

None.

\section{References}

1 Wolfe JB. Report of the Committee on Nomenclature of the American Society for the Study of Arterio-sclerosis. Circulation 1955;12:1065

2 Kerr GS, Hallahan CW, Giordano J, et al. Takayasu arteritis. Ann Intern Med 1994;120(11):919-929

3 Panja M, Kar AK, Dutta AL, Chhetri M, Kumar S, Panja S. Cardiac involvement in non-specific aorto-arteritis. Int J Cardiol 1992;34(3):289-295

4 Horimoto M, Igarashi K, Aoi K, Okamoto K, Takenaka T. Unilateral diffuse pulmonary artery involvement in Takayasu's arteritis associated with coronary-pulmonary artery fistula and bronchial-pulmonary artery fistula: a case report. Angiology 1991;42(1):73-80

5 Ercan E, Tengiz I, Yakut N, Gurbuz A, Bozdemir H, Bozdemir G. Takayasu's arteritis with multiple fistulas from three coronary arteries to lung paranchima. Int J Cardiol 2003;88(2-3):319-320

6 Liberthson RR, Sagar K, Berkoben JP, Weintraub RM, Levine FH. Congenital coronary arteriovenous fistula. Report of 13 patients, review of the literature and delineation of management. Circulation 1979;59(5):849-854

7 Arend WP, Michel BA, Bloch DA, et al. The American College of Rheumatology 1990 criteria for the classification of Takayasu's arteritis. Arthritis Rheum 1990;33:11291134

8 Wilcox WD, Neal MJ, Alpert BS, Taylor AB, Dooley KJ. Localized occurrence of congenital coronary artery fistula in the southeast United States. Am J Cardiol 1986;57(4):361-363

9 Zenooz NA, Habibi R, Mammen L, Finn JP, Gilkeson RC. Coronary artery fistulas: CT findings. Radiographics 2009; 29(3):781-789

10 Said SA, Lam J, van der Werf T. Solitary coronary artery fistulas: a congenital anomaly in children and adults. A contemporary review. Congenit Heart Dis 2006;1(3):63-76

11 Sharma S, Kamalakar T, Rajani M, Talwar KK, Shrivastava S. The incidence and patterns of pulmonary artery involvement in Takayasu's arteritis. Clin Radiol 1990;42(3):177-181

12 Yamato M, Lecky JW, Hiramatsu K, Kohda E. Takayasu arteritis: radiographic and angiographic findings in 59 patients. Radiology 1986;161(2):329-334

13 Lupi E, Sánchez G, Horwitz S, Gutierrez E. Pulmonary artery involvement in Takayasu's arteritis. Chest 1975;67(1):69-74

14 Karabay KO, Altuntas E, Uysal E, Ozkara A, Aytekin V. Takayasu arteritis with multiple coronary artery fistulas to bronchial arteries. Int J Angiol 2016;25(5):e1-e3 